\title{
Tetraether membrane lipids of Candidatus "Aciduliprofundum boonei", a cultivated obligate thermoacidophilic euryarchaeote from deep-sea hydrothermal vents
}

\author{
Stefan Schouten · Marianne Baas · Ellen C. Hopmans • \\ Anna-Louise Reysenbach · Jaap S. Sinninghe Damsté
}

Received: 11 June 2007 / Accepted: 28 August 2007/Published online: 28 September 2007

(C) Springer 2007

\begin{abstract}
The lipid composition of Candidatus "Aciduliprofundum boonei", the only cultivated representative of archaea falling in the DHVE2 phylogenetic cluster, a group of microorganisms ubiquitously occurring at hydrothermal vents, was studied. The predominant core membrane lipids in this thermophilic euryarchaeote were found to be composed of glycerol dibiphytanyl glycerol tetraethers (GDGTs) containing 0-4 cyclopentyl moieties. In addition, GDGTs with an additional covalent bond between the isoprenoid hydrocarbon chains, so-called H-shaped GDGTs, were present. The latter core lipids have been rarely reported previously. Intact polar lipid analysis revealed that they predominantly consist of GDGTs with a phospho-glycerol headgroup.
\end{abstract}

Keywords Aciduliprofundum boonei - DHVE2 cluster · Glycerol dialkyl glycerol tetraethers - Thermoacidophile

\section{Introduction}

Deep-sea hydrothermal vents are unique environments, which are thought to represent models for both the origin of life on Earth and exploration of life on other planets. These

Communicated by J. N. Reeve.

S. Schouten $(\bowtie) \cdot$ M. Baas · E. C. Hopmans · J. S. S. Damsté Department of Marine Biogeochemistry and Toxicology, Royal Netherlands Institute for Sea Research, PO Box 59, 1790 AB Den Burg, Texel, The Netherlands e-mail: schouten@nioz.nl

A.-L. Reysenbach

Department of Biology, Portland State University, Portland, OR 97201, USA vents contain ecosystems, which are predominantly fueled by geochemical energy and are host to many newly described free-living microbes, which are often associated with actively venting porous deep-sea vent deposits or "chimneys". The steep chemical and thermal gradients within the walls of these deposits provide a wide range of microhabitats for microorganisms with suitable conditions for aerobic and anaerobic thermophiles and mesophiles (e.g. McCollom and Schock 1997). Indeed, both culturedependent and -independent approaches have exposed a vast diversity of Bacteria and Archaea associated with deep-sea vent deposits (e.g. Reysenbach and Shock 2002; Schrenk et al. 2003). Numerous Archaea have been isolated from these deposits but few of these are found in environmental 16S rRNA gene clone libraries and most detected environmental clones in these environments have no representatives available in pure culture.

In particular, one archaeal lineage is widespread at deepsea vents, namely the "deep-sea hydrothermal vent euryarchaeotic" lineage DHVE2, and is frequently associated with actively venting sulphide deposits (e.g. Nercessian et al. 2003; Hoek et al. 2003; Reysenbach and Shock 2002; Takai et al. 1999, 2001). In some cases it has been reported as the most dominant clone type in archaeal clone libraries (Hoek et al. 2003), yet the physiology of these organisms was unclear. Recently, Reysenbach et al. (2006) isolated and cultivated a member of the DHVE2 phylogenetic DHVE2 cluster, Candidatus "Aciduliprofundum boonei", and showed it to be an obligate thermoacidophilic sulphur and iron reducing heterotroph capable of growing from $\mathrm{pH} 3.3$ to 5.8 and between 60 and $75^{\circ} \mathrm{C}$. This provided the first evidence that thermoacidophiles may be key players in sulphur and iron cycling at deep-sea vents.

Archaea are not only unique in their 16S rRNA phylogenetic position in the tree of life, but also synthesize 
specific membrane lipids. Analysis of cultivated hyperthermophilic Archaea showed that their membrane is predominantly composed of isoprenoid glycerol dibiphytanyl glycerol tetraethers (GDGTs) with additional cyclopentyl moieties (e.g. Structures I-VI in Fig. 1). The structural differences from diacyl membrane lipids of nonthermophilic Eukarya and Bacteria, i.e. ether bonds and the formation of a monolayer rather than a bilayer, have been suggested to contribute to the stability of membranes of hyperthermophiles at high temperatures and low pH (e.g. De Rosa and Gambacorta 1988; van den Vossenberg et al. 1998; Macalady et al. 2004). This suggests that members of the DHVE2 cluster may be also synthesizing GDGT membrane lipids. Therefore, in this study we analyzed the lipid composition of the only cultivated representative of DHVE2, Aciduliprofundum boonei, and examined both the core GDGT lipid composition as well as its intact polar lipid composition.

\section{Materials and methods}

Culture conditions

Aciduliprofundum boonei was grown as previously described (Reysenbach et al. 2006). Cells were harvested at late $\log$ phase of growth. Cell pellets were freeze-dried and stored frozen until analysis.

Core lipid analysis

Cell material (74 mg dry weight) of A. boonei was hydrolyzed by refluxing in ca. $4 \mathrm{ml} 1 \mathrm{M} \mathrm{KOH}$ in methanol for $1 \mathrm{~h}$. The $\mathrm{pH}$ of the hydrolyzed extract was adjusted to pH 3 using $2 \mathrm{M} \mathrm{HCl} /$ methanol $(\mathrm{MeOH}) 1 / 1(\mathrm{v} / \mathrm{v})$ and transferred to a separatory funnel. The residual cell material was washed subsequently with $2 \mathrm{ml} \mathrm{MeOH} /$ water
Fig. 1 HPLC/APCI/MS base peak chromatogram of GDGT core lipids in extract released after base hydrolysis (using $\mathrm{KOH} /$ methanol mixture) of the cell material of Candidatus "Aciduliprofundum boonei". Inset shows the atmospheric pressure chemical ionization mass spectrum of GDGT VII. Note that the position of the covalent bond between the isoprenoid hydrocarbon chains in GDGTs VII-XI is tentative (Morii et al. 1998)
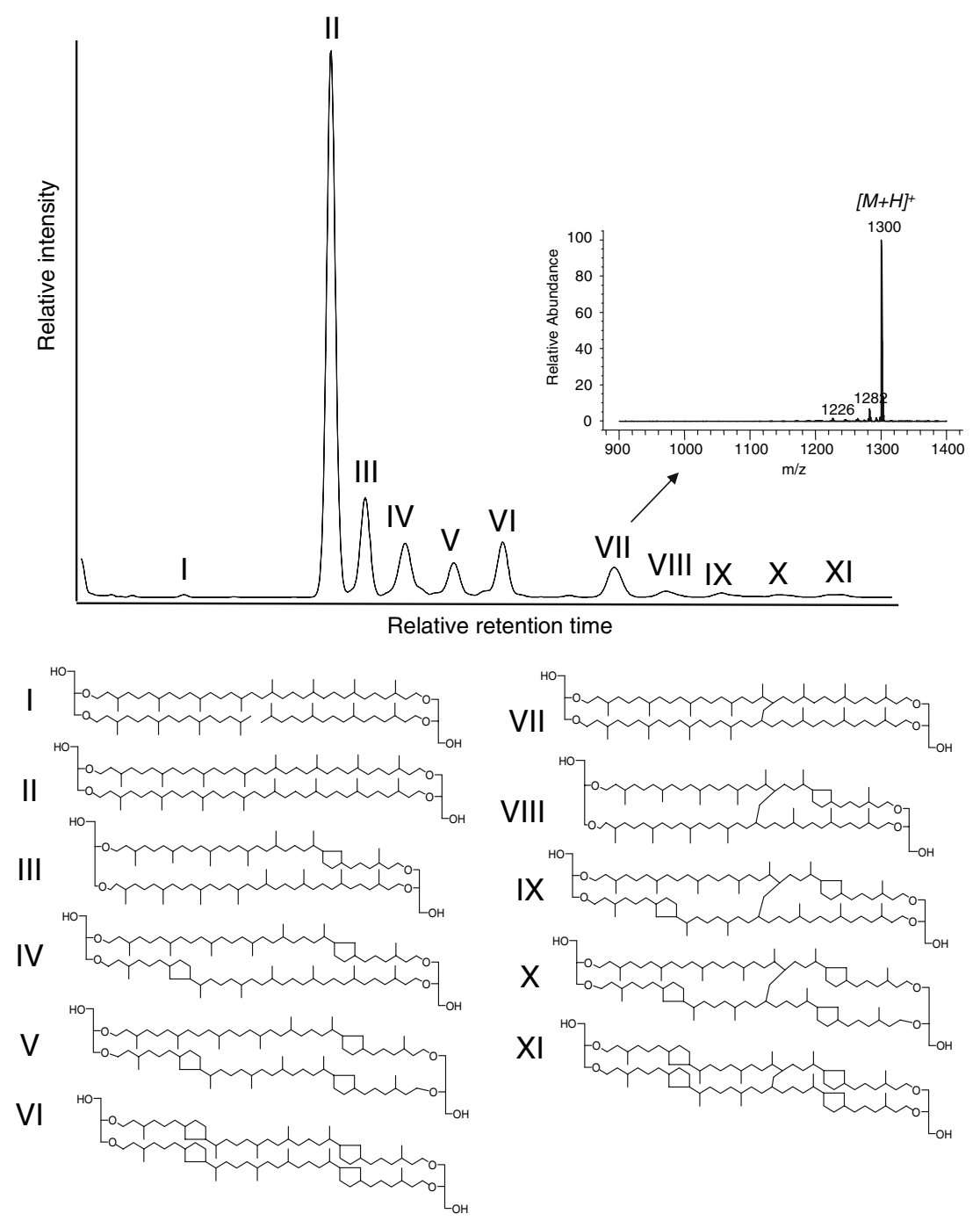
1/1 (v/v), $\mathrm{MeOH}$ and dichloromethane (DCM, three times). The washings and $6 \mathrm{ml}$ bidistilled water were added to the separatory funnel, which was shaken vigorously. The DCM layer was removed and the residual water/MeOH layer was re-extracted two times using DCM. The combined DCM layers were dried using a sodium sulfate column to yield the hydrolyzed extract. The extract released upon hydrolysis was condensed by rotary evaporation. Elemental sulphur was removed with activated $\mathrm{Cu}$. The extract was methylated by diazomethane and filtered over a silicagel column using ethyl acetate. One aliquot of the eluate was silylated using BSTFA (with $1 \%$ TMCS) in pyridine (1/1, $\mathrm{v} / \mathrm{v}$ ) for $20 \mathrm{~min}$ at $60^{\circ} \mathrm{C}$ and subsequently analyzed by gas chromatography (GC) and $\mathrm{GC} /$ mass spectrometry (GC/ MS). Another aliquot of the eluate was dissolved in hexane/isopropanol (99:1, v/v), ultrasonicated and filtered using a PTFE $0.45-\mu \mathrm{m}$ filter prior to analysis for GDGTs on the high performance liquid chromatography (HPLC)/ MS.

Part of the GDGT fraction was hydrogenated by dissolving it in ethyl acetate, adding a small amount of $\mathrm{PtO}_{2}$ and a drop of acetic acid and bubbling hydrogen for $1 \mathrm{~h}$. Subsequently, the solution was stirred overnight and filtered over a small pipette column containing $\mathrm{Na}_{2} \mathrm{CO}_{3}$ (top) and $\mathrm{MgSO}_{4}$ (bottom). Ethyl acetate was evaporated and the hydrogenated GDGT fraction was dissolved in hexane/ isopropanol (99:1, v/v), ultrasonicated and analyzed using HPLC/MS.

A second aliquot of the GDGT fraction was separated using HPLC with a preparative device (Foxy jr) and the fractions collected were analyzed by flow injection analysis mass spectrometry (Smittenberg et al. 2002). The fractions containing the unusual GDGT (see text below) were combined and treated with $\mathrm{HI}$ and $\mathrm{LiAlH}_{4}$ to release the ether bound hydrocarbon skeletons (Schouten et al. 1998). This fraction was analyzed by GC and GC/MS.

The total extract and the GDGT fractions treated with $\mathrm{HI} / \mathrm{LiAlH}_{4}$ were analyzed by GC/MS using a Finnigan Trace GC ultra coupled to a Finnigan Trace DSQ mass spectrometer. A fused silica capillary column $(25 \mathrm{~m} \times 0.32 \mathrm{~mm})$ coated with CP-Sil 5 (film thickness $0.12 \mu \mathrm{m}$ ) was used with helium as carrier gas. The oven was programmed at a starting (injection) temperature of $70^{\circ} \mathrm{C}$, which rose to $130^{\circ} \mathrm{C}$ at $20^{\circ} \mathrm{C} / \mathrm{min}$ and then to $320^{\circ} \mathrm{C}$ at $4^{\circ} \mathrm{C} / \mathrm{min}$, at which it was maintained for $20 \mathrm{~min}$. The column was directly inserted into the electron impact ion source of the DSQ quadrupole mass spectrometer, scanning a mass range of $m / z, 50-800$ at three scans per second and with an ionization energy of $70 \mathrm{eV}$.

HPLC/MS analyses were performed according to Hopmans et al. (2000). Analyses were performed using an Agilent (Palo-Alto, CA, USA) 1100 series LC-MSD SL equipped with an auto-injector and Chemstation chromatography manager software. Separation was achieved on a Prevail Cyano column $(2.1 \times 150 \mathrm{~mm}$, $3 \mu \mathrm{m}$; Alltech, Deerfield, IL, USA), maintained at $30^{\circ} \mathrm{C}$. Injection volumes were $10 \mu \mathrm{l}$. GDGTs were eluted isocratically with $99 \% \mathrm{~A}$ and $1 \% \mathrm{~B}$ for $5 \mathrm{~min}$, followed by a linear gradient to $1.8 \% \mathrm{~B}$ in $45 \mathrm{~min}$, where $\mathrm{A}=$ hexane and $\mathrm{B}=$ isopropanol. Flow rate was $0.2 \mathrm{ml} / \mathrm{min}$. After each analysis the column was cleaned by back-flushing hexane/ isopropanol $(90: 10, \mathrm{v} / \mathrm{v})$ at $0.2 \mathrm{ml} / \mathrm{min}$ for $10 \mathrm{~min}$. Detection was achieved using atmospheric pressure positive ion chemical ionization mass spectrometry (APCI-MS) of the eluent. Conditions for the HP 1100 APCI-MSD SL were as follows: nebulizer pressure $60 \mathrm{psi}$, vaporizer temperature $400^{\circ} \mathrm{C}$, drying gas $\left(\mathrm{N}_{2}\right)$ flow $6 \mathrm{l} / \mathrm{min}$ and temperature $200^{\circ} \mathrm{C}$, capillary voltage $-3 \mathrm{kV}$, corona $5 \mu \mathrm{A}(\sim 3.2 \mathrm{kV})$. GDGTs were detected by mass scanning from $\mathrm{m} / \mathrm{z}$ 9501,450 .

Intact polar lipid analysis

For analysis of the intact polar lipids, cell material of A. boonei were extracted using a modified Bligh-Dyer procedure (Bligh and Dyer 1959). To an aliquot of the cell material, a solvent mixture of phosphate-buffer $(0.05 \mathrm{M}$, $\mathrm{pH}$ 7.4)/MeOH/DCM 0.8/2/1 (v/v) was added. The mixture was sonicated for $10 \mathrm{~min}$ after which DCM and phosphate buffer were added to a volume ratio of $0.9 / 1 / 1$. After centrifuging (2,500 rpm, $5 \mathrm{~min}$ ) the DCM layer was collected. The residue was re-extracted twice following the same procedure. The combined DCM layers were concentrated by evaporating solvent under a $\mathrm{N}_{2}$ stream, dried with $\mathrm{Na}_{2} \mathrm{SO}_{4}$ and the solvent was removed under a $\mathrm{N}_{2}$ stream. The residue was dissolved in a mixture of hexane/isopropanol (79:21, v/v), ultrasonicated and filtered using a RC $0.45-\mu \mathrm{m}$ filter prior to analysis on the HPLC/electrospray ionization (ESI)-MS.

Intact core lipids were analyzed according to Sturt et al. (2004) with some modifications (Boumann et al. 2006). An Agilent 1100 series LC (Agilent, San Jose, CA, USA) was used, equipped with thermostatted autoinjector and column oven, coupled to a Thermo TSQ Quantum Ultra EM triple quadrupole mass spectrometer equipped with an Ion Max source with ESI probe (Thermo Electron Corporation, Waltham, MA, USA). Separation was achieved on an Inertsil diol column $(250 \mathrm{~mm} \times 2.1 \mathrm{~mm}, 5 \mu \mathrm{m}$ particles; Alltech Associates Inc., Deerfield, IL, USA) maintained at $30^{\circ} \mathrm{C}$. The following linear gradient was used with a flow rate of $0.2 \mathrm{~mL} \mathrm{~min}^{-1}$ : $100-35 \% \mathrm{~A}: 65 \% \mathrm{~B}$ over $45 \mathrm{~min}$, maintained for $20 \mathrm{~min}$, then back to $100 \% \mathrm{~A}$ for $20 \mathrm{~min}$ to re-equilibrate the column, where $\mathrm{A}=$ hexane/2-propanol/ formic acid/14.8 $\mathrm{M} \mathrm{NH}_{3 \mathrm{aq}}$ ratios 79:20:0.12:0.04 (v/v/v/v) and $\mathrm{B}=2$-propanol/water/formic acid/14.8 $\mathrm{M} \mathrm{NH}_{3 \text { aq }}$ ratios 
88:10:0.12:0.04 (v/v/v/v). For MS detection, source parameters were optimized using loop injections of standard phospholipids (1,2-dipalmitoyl-sn-glycero-3phosphocholine, 1,2-dipalmitoyl-sn-glycero-3-phosphoethanolamine, 1,2-dipalmitoyl-sn-glycero-3-phospho-L-serine, 1,2-dipalmitoyl-sn-glycero-3-phospho-rac-glycerol, 1,2dipalmitoyl-sn-glycero-3-phosphate, and soya L- $\alpha$-phosphatidylinositol, Avanti Polar Lipids, Alabaster, AL, USA) into a stream of $0.2 \mathrm{ml} / \mathrm{min}$ of eluent A. ESI settings were as follows: capillary temperature $250^{\circ} \mathrm{C}$, sheath gas $\left(\mathrm{N}_{2}\right)$ pressure 49 (arbitrary units, auxiliary gas) $\left(\mathrm{N}_{2}\right)$ pressure 21 (arbitrary units), spray voltage $4.2 \mathrm{kV}$, and source CID$14 \mathrm{~V}$. Mass detection was achieved on the first MS scanning from $\mathrm{m} / \mathrm{z}, 000$ to 2,000 .

\section{Results and discussion}

Core lipids

GC/MS analysis of the lipid extract of hydrolyzed cell material of $A$. boonei did not reveal substantial amounts of dialkyl glycerol ethers, such as archaeol, suggesting that it does not accumulate these compounds in substantial amounts. By contrast, HPLC/APCI-MS analysis of the fraction revealed the abundant presence of a range of GDGTs (Fig. 1) suggesting that archaeol is effectively transformed into GDGTs. The main compound was GDGT II, containing no cyclopentyl moieties, with lower amounts of GDGTs III-VI, containing 1-4 cyclopentyl moieties, and small amounts of GDGT I. These GDGTs are well known compounds of thermoacidophilic Archaea in both the euryarchaeotal and crenarchaeotal groups (cf. De Rosa and Gambacorta 1988; Koga and Morii 2005). Cultivated species of the Order Thermoplasmatales, the closest phylogenetic relatives to A. boonei (Reysenbach et al. 2006), also contain GDGTs II-VI as their dominant membrane lipids (DeRosa and Gambacorta 1988; Uda et al. 2004; Macalady et al. 2004).

Besides GDGTs I-VI, another group of later-eluting compounds were present in the HPLC/MS chromatogram with mass spectra (insert Fig. 1) characteristic of GDGTs, i.e. a base peak likely representing the $[\mathrm{M}+\mathrm{H}]^{+}$ions and fragment ions with losses of $18 \mathrm{Da}$ (hydroxyl group as water) and $74 \mathrm{Da}$ (part of the glycerol moiety) (Hopmans et al. 2000). The main compound had a base peak ion of 1,300 with more minor compounds having base peak ions of 1,298, 1,296, 1,294 and 1,292, respectively. Remarkably, the distribution of these compounds is similar to that of GDGTs II-VI and their base peak ions are all 2 Da lower than their GDGT counterparts. This suggests that these compounds are GDGTs with an additional double bond or an additional cyclic moiety. To investigate the first hypothesis, we hydrogenated the polar fraction to remove any double bonds. However, subsequent analysis by HPLC/MS revealed no changes in GDGT composition, suggesting that these compounds did not contain any double bonds. The unknown GDGT was subsequently isolated by preparative HPLC and treated with HI/LiAlH to release the hydrocarbon skeletons (cf. Schouten et al. 1998). GC/MS analysis revealed, however, no distinct peaks suggesting that the hydrocarbon skeletons of the unknown GDGT do not consist of regular $\mathrm{C}_{40}$ isoprenoids. An explanation for these results can be that the hydrocarbon skeletons are covalently linked to each other, thereby forming a $\mathrm{C}_{80}$ hydrocarbon after ether-cleavage. The molecular weight of such a component is too high to elute over a regular GC column. Examples of such GDGTs have been previously reported, i.e. the so-called H-shaped GDGT (Morii et al. 1998) that contains a covalent bond between the two isoprenoid chains. We, therefore, analyzed a pure GDGT VII standard obtained from Methanothermus fervidus (a kind gift of Dr. T. Eguchi) by HPLC/MS. The mass spectrum of this standard was identical to that of the major unknown GDGT in $A$. boonei and co-injection revealed that they have identical retention times, thus unambiguously identifying this compound as GDGT VII. This suggests that the other later eluting GDGTs are also H-shaped isoprenoid GDGTs but with additional cyclopentyl moieties, i.e., GDGTs VII-XI. It must be noted that the exact position of the covalent bond in GDGT VII is tentative (Morii et al. 1998) and, thus, also the position of the covalent bonds in GDGTs VII-XI. Lutneas et al. (2007) recently determined the structure of structurally similar isoprenoid tetracids in which the covalent bond was between two methyl groups rather than between a methyl group and the aliphatic chain as suggested by Morii et al. (1998). Hence, future structural analyses are needed to unambiguously establish the position of the covalent bond.

$\mathrm{H}$-shaped isoprenoid GDGTs have been only rarely reported in cultivated archaea. GDGT VII was first identified in the hyperthermophilic methanogen Methanothermus fervidus (Morii et al. 1998). They were further identified by fast atom bombardment-MS in 4 out of 17 species of the Order Thermococcales (Sugai et al. 2004). Until now, they have only been identified in hyperthermophilic euryarchaeota growing at neutral $\mathrm{pH}$ with optimal growth temperatures $>80^{\circ} \mathrm{C}$. The identification of $\mathrm{H}$ shaped GDGTs in A. boonei now shows that these compounds are also synthesized at lower growth temperatures $\left(70^{\circ} \mathrm{C}\right)$ and more acidic $\mathrm{pH}(4.5)$. The functional role of these GDGTs is not clear. Possibly, the introduction of a covalent cross-link between the alkyl chains will reinforce the strength of the monolayer membrane to protect the cell against membrane lysis at these high temperatures. The similar relative distribution of GDGTs II-VI compared to 
Fig. 2 HPLC/ESI/MS base peak chromatogram of intact polar lipids in Bligh Dyer extract of cell material of Candidatus "Aciduliprofundum boonei". Inset shows the electrospray ionization mass spectrum of the major peak in the chromatogram with tentative structural identification

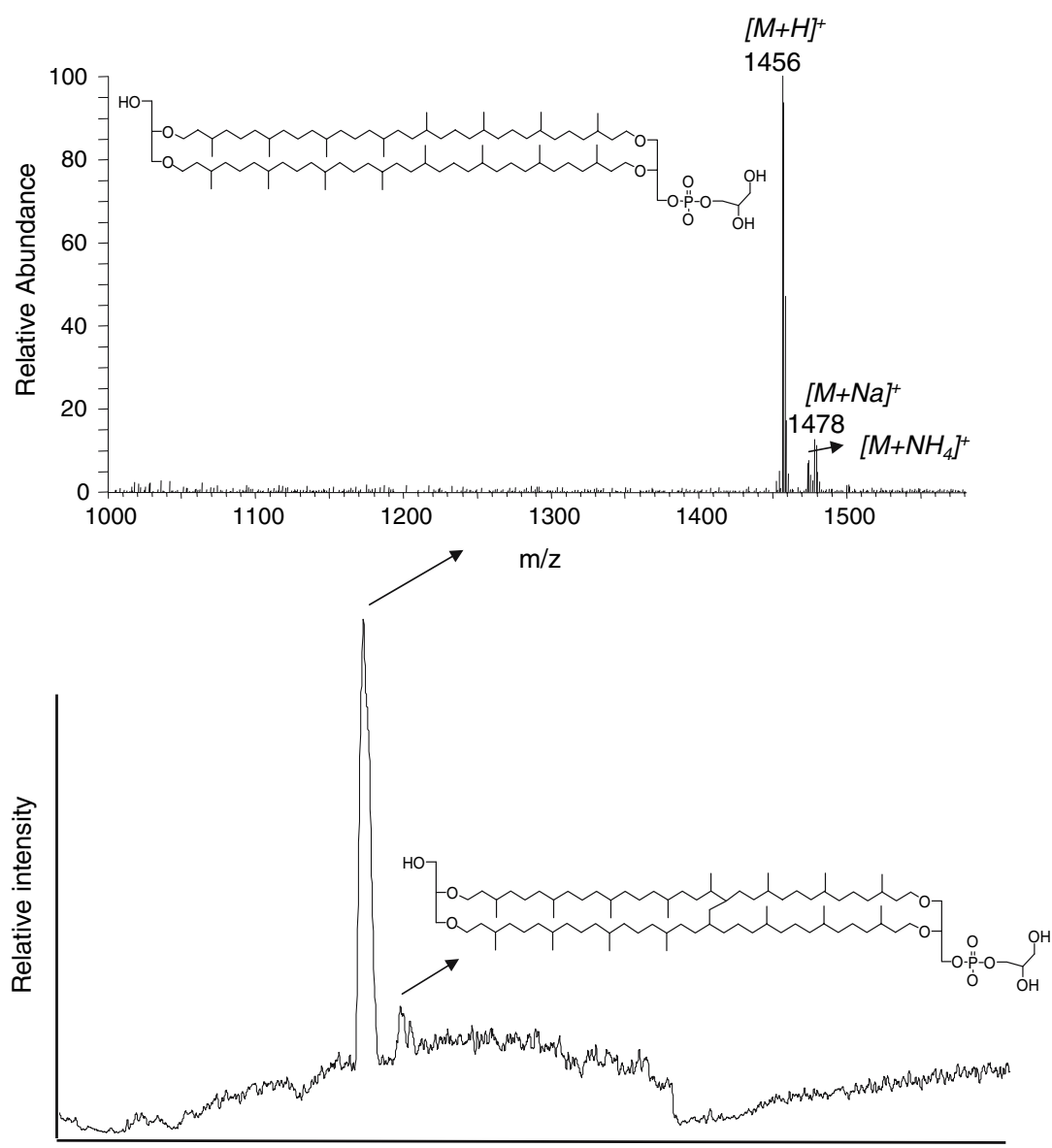

Relative retention time
GDGTs VII-XI in A. boonei tentatively suggests a possible biosynthetic link, i.e., the latter GDGTs may be synthesized from GDGTs II-VI.

Intact polar lipids

HPLC/ESI-MS analysis of the Bligh-Dyer extract of cell material of $A$. boonei revealed one major peak and one minor peak (Fig. 2). The mass spectrum of the major peak is dominated by an ion at $m / z, 4,456$ with minor ions 15 and 22 Da higher (insert Fig. 2). These latter ions are typical product of ammonia and sodium adductions, respectively, to protonated molecules in the ESI interface and suggests that the ion at $m / z, 1,456$ represents the $[\mathrm{M}+\mathrm{H}]^{+}$ion (cf. Sturt et al. 2004). As the major core lipid is GDGT II with a $[\mathrm{M}+\mathrm{H}]^{+}$ion of 1,302 , it suggests that the head group has a mass of 154 Da in agreement with a phospho-glycerol (PG) moiety (Sturt et al. 2004). The mass spectrum of the minor peak eluting slightly later is similar to that of the major peak except that all ions are shifted 2 Da lower. In view of the results obtained with the core lipids it suggests that this peak represents H-shaped GDGTs with a PG head group.
PG moieties are not uncommon as head groups of GDGTs in hyperthermophilic Archaea, though they are usually accompanied by a hexose head group attached to the other glycerol moiety (Koga and Morii 2005 and references cited therein). However, these compounds were not detected in A. boonei although we cannot exclude that they might be present in minor amounts.

\section{Conclusions}

Our analysis of lipid composition of Candidatus "Aciduliprofundum boonei", the only cultivated representative of archaea falling in the DHVE2 phylogenetic cluster, showed it to be composed of GDGTs containing 0-4 cyclopentyl moieties. Surprisingly, GDGTs with an additional covalent bond between the isoprenoid hydrocarbon chains, the socalled H-shaped GDGTs were also present in substantial amounts. These GDGTs mainly occurred with a phosphoglycerol headgroup in the membrane of A. boonei.

Acknowledgments We thank Dr. T. Eguchi for the kind gift of the GDGT VII standard. This work was partially supported by an 
instrument grant from NWO to ECH and an NSF grant to ALR (OCE0242038).

\section{References}

Bligh EG, Dyer WJ (1959) A rapid method of total lipid extraction and purification. Can J Biochem Physiol 37:911-917

Boumann HA, Hopmans EC, Op Den Camp H, Strous M, Jetten MSM, Sinninghe Damsté JS, Schouten S (2006) Ladderane phospolipids in anammox bacteria comprise phosphocholine and phosphoethanolamine headgroups. FEMS Microbiol Lett 258:297-304

De Rosa M, Gambacorta A (1988) The lipids of archaeabacteria. Prog Lipid Res 27:153-175

Hoek J, Banta AB, Hubler RF, Reysenbach A-L (2003) Microbial diversity of a deep-sea hydrothermal vent biofilm from Edmond vent field on the Central Indian Ridge. Geobiology 1:119-127

Hopmans EC, Schouten S, Pancost RD, van der Meer MTJ, Sinninghe Damsté JS (2000) Analysis of intact tetraether lipids in archaeal cell material and sediments by high performance liquid chromatography/atmospheric pressure chemical ionization mass spectrometry. Rap Commun Mass Spectrom 14:585-589

Koga Y, Morii H (2005) Recent advances in structural research on ether lipids from archaea including comparative and physiological aspects. Biosci Biotechnol Biochem 69:2019-2034

Lutnaes BF, Krane J, Smith BE, Rowland SJ (2007) Structure elucidation of $\mathrm{C}_{80}, \mathrm{C}_{81}$ and $\mathrm{C}_{82}$ isoprenoid tetraacids responsible for naphthenate deposition in crude oil production. Org Biomol Chem 5:1873-1877

Macalady JL, Vestling MM, Baumler D, Boekelheide N, Kaspar CW, Banfield JF (2004) Tetraether-linked membrane monolayers in Ferroplasma spp: a key to survival in acid. Extremophiles 8:411-419

McCollom TM, Shock EL (1997) Geochemical constraints on chemolithoautotrophic metabolism by microorganisms in seafloor hydrothermal systems. Geochim Cosmochim Acta 61:4375-4391

Morii H, Eguchi T, Nishihara M, Kakinuma K, König H, Koga Y (1998) A novel ether core lipid with $\mathrm{H}$-shaped $\mathrm{C}_{80^{-}}$isoprenoid hydrocarbon chain from the hyperthermophilic methanogen Methanothermus fervidus. Biochim Biophys Acta 1390:339-345
Nercessian O, Reysenbach A-L, Prieur D, Jeanthon C (2003) Compositional changes in archaeal communities associated with deep-sea hydrothermal vent samples from the East Pacific Rise $(13 \mathrm{~N})$. Environ Microbiol 5:492-502

Reysenbach AL, Shock EL (2002) Merging genomes with geochemistry in hydrothermal ecosystems. Science 296:1077-1082

Reysenbach AL, Liu Y, Banta AB, Beveridge TJ, Kirshtein JD, Schouten S, Tivey MK, Von Damm K, Voytek MA (2006) Isolation of a ubiquitous obligate thermoacidophilic archaeon from deep-sea hydrothermal vents. Nature 442:444-447

Schouten S, Hoefs MJL, Koopmans MP, Bosch H-J, Sinninghe Damsté JS (1998) Structural identification, occurrence and fate of archaeal ether-bound acyclic and cyclic biphytanes and corresponding diols in sediments. Org Geochem 29:1305-1319

Schrenk MO, Kelley DS, Delaney JR, Baross JA (2003) Incidence and diversity of microorganisms within the walls of an active deep-sea sulfide chimney. Appl Environ Microbiol 69:35803592

Smittenberg RH, Hopmans EC, Schouten S, Sinninghe Damsté JS (2002) Rapid isolation of biomarkers for compound specific radiocarbon dating using HPLC and FIA-APCI/MS. J Chrom A 978:129-140

Sturt HF, Summons RE, Smith K, Elvert M, Hinrichs KU (2004) Intact polar membrane lipids in prokaryotes and sediments deciphered by high-performance liquid chromatography/electrospray ionization multistage mass spectrometry-new biomarkers for biogeochemistry and microbial ecology. Rapid Commun Mass Spectrom 18:617-28

Sugai A, Uda I, Itoh Y, Itoh YH, Itoh T (2004) The core lipid composition of the 17 strains of hyperthermophilic archaea, Thermococcales. J Oleo Sci 53:41-44

Takai K, Horikoshi K (1999) Genetic diversity of archaea in deep-sea hydrothermal vent environments. Genetics 152:1285-1297

Takai K, Komatsu T, Inagari F, Horikoshi K (2001) Distribution of Archaea in a black smoker chimney structure. Appl Environ Microbiol 67:3618-3629

Uda I, Sugai A, Itoh YH, Itoh T (2004) Variation in molecular species of core lipids from the order thermoplasmales strains depends on growth temperature. J Oleo Sci 53:399-404

Van den Vossenberg JLCM, Driessen AJM, Konings WN (1998) The essence of being extremophilic: the role of unique archaeal membrane lipids. Extremophiles 2:163-170 